\title{
Anterior Tibial Artery Terminating as Tarsal arteries
}

\author{
Dr. Jyoti Kulkarni ${ }^{1}$, Dr. Vaishali Paranjpe ${ }^{2}$, Dr. Vatsalaswamy ${ }^{3}$ \\ ${ }^{1,2,3}$ (Department of Anatomy, Dr. DY Patil Medical College/Dr. DY Patil University, Pimpri, Pune, India)
}

\begin{abstract}
The anterior tibial artery terminated into medial tarsal and lateral tarsal branches. Dorsalis pedis artery was very thin arising as a branch from medial tarsal artery. The first and second dorsal metatarsal arteries were seen arising from lateral tarsal artery. Arcuate artery was absent. Knowledge of vascular anatomy of foot is essential for arterial reconstruction flap surgeries of the foot. This can avoid amputation of foot in cases of arterial trauma like thromboangitis obliterans, industrial automobile accidents, diabetes and severe ischaemia of lower limb.
\end{abstract}

Key words: Anterior tibial artery, Arcuate artery, Dorsalis pedis artery, Medial Tarsal artery, Lateral Tarsal artery.

\section{Introduction}

During routine dissection of a male cadaver in the department of Anatomy, Dr. D.Y. Patil Medical college, Pimpri,Pune, a variation in the termination pattern of anterior tibial artery was found. The artery was carefully cleaned and dissected. Normally Anterior tibial artery (A) at the level of ankle joint gives medial and lateral malleolar branches (B \& C). Then it continues as a dorsalis pedis artery (D) lying deep to inferior extensor retinaculum and distal to ankle joint (Fig1). Medial and lateral tarsal arteries arise from dorsalis pedis as it crosses the navicular. Here it lies deep to extensor digitorum brevis. Dorsalis pedis then enters the sole to complete the plantar arch after giving arcuate branch and first dorsal metatarsal branch ${ }^{[4]}$. The most preferred site for palpation of dorsalis pedis artery is against the navicular bone. However it can be palpated from midpoint between the malleoli to the proximal end of first intermetatarsal space. The dorsalis pedis pulse is evaluated during physical examination of the peripheral vascular system. Dorsalis pedis pulse is palpated with the feet slightly dorsiflexed. The pulse is usually easy to palpate because the dorsal ateries of the foot are subcutaneous and pass along a line from the extensor retinaculum to a point just lateral to the extensor hallucis tendon. A diminished or absent dorsalis pedis pulse usually suggest vascular insufficiency. However some healthy individuals may have a congenitally non palpable dorsalis pedis pulse ${ }^{[5]}$. In these cases there is a variation in the termination pattern of anterior tibial artery. The anterior tibial artery may fail to grow more than a short way down the leg. In about $14 \%$ of cases the dorsalis pedis artery may be replaced by a perforating branch of peroneal artery or perforating branch of posterior tibial artery ${ }^{[6]}$.

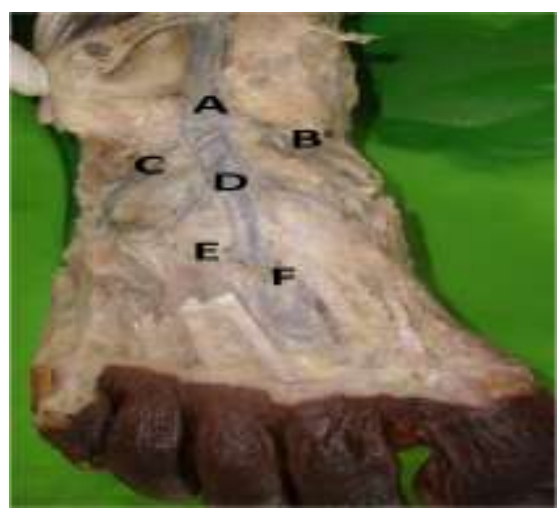

Figure 1. Dorsum of Right Foot

A. Anterior Tibial artery

B. Medial Tarsal artery

C. Lateral Tarsal artery

D. Dorsalis Pedis artery

E. Arcuate artery

F. First dorsal metatarsal arterv

\section{Case Report}

In the present case the anterior tibial artery (A) terminated into medial tarsal (B) and lateral tarsal branches (C). Dorsalis pedis artery (D) was seen as a very thin branch of medial tarsal artery. The medial malleolar and lateral malleolar branch $(\mathrm{G} \& \mathrm{H})$ were found to be the branches from medial and lateral tarsal arteries respectively. The first and second dorsal metatarsal arteries (E \& F) were seen arising from lateral tarsal artery. Arcuate artery was found to be absent (Fig.2). 


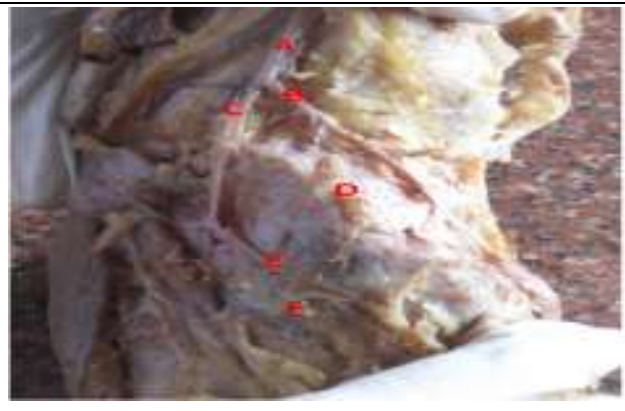

Figure 2. Dorsum of Right

Foot

A. Anterior Tibial artery

B. Medial Tarsal artery

C. Lateral Tarsal artery

D. Dorsalis pedis artery

E. First dorsal metatarsal artery

F. Second dorsal metatarsal artery

\section{Discussion}

- Variations in the blood vessels and their anomalous course can be attributed to their development. Tiny blood vessels derived from the blood islands in the $3^{\text {rd }} \& 4^{\text {th }}$ week of development merge with each other and form a continuous network from which buds grow out, canalize and form new vessels. New vessels of neighborhood areas form a closed network. Depending on the functional dominance, some vessels regress and others diverge in the mode of origin and course from the principal vessels ${ }^{[3]}$.

- Dorsalis pedis artery is the largest artery distal to ankle joint. It is used for pedal revascularisation surgeries. Vascular anatomy is an indispensible parameter for flap selection in reconstructive surgeries in order to ensure the viability of flap. Keeping this in mind a detailed study of termination of anterior tibial artery is now being undertaken, followed by study of vascular anatomy of foot.

- Knowledge of individual variations of the foot arteries is studied by arteriography before using extensor digitorum brevis or the skin of the dorsum of the foot as a flap ${ }^{[1]}$.

- Study of variation is also useful in deciding whether the absence of pulse in dorsalis pedis artery is due to thrombosis of the vessel or its variant course or its absence ${ }^{[2]}$. Examination of pedal pulse is a useful clinical tool to evaluate peripheral circulation. Although a correlation between the ability to palpate pulses and the degree of systolic perfusion pressure has been established controversy surrounds its accuracy ${ }^{[7] .}$

- Variation in the interpretation of results of investigations related to blood vessels is due to discrepancies related to arterial size, variation, amount of subcutaneous fat, edema or neurovascular diabetic changes ${ }^{[8]}$.

\section{References}

[1] EMEI - Saeed et al - Anatomical study of the Dorsalis pedis artery and its surgical importance in reconstructive surgeries Allexandria Bulletin pg- 557 to 571

[2] VijayaLakshmi S, Gunapriya Raghunat, Varsha Shenoy Anatomical study of dorsalis pedis artery and its clinical correlations - Journal of clinical and diagnostic research 2011 April, Vol - 5 (2) : $287-290$

[3] Sadler T. W (1985) in Langman's Medical Embryology $5^{\text {th }}$ edition Wiliam and Wilkins; 68-69

[4] Williams, Peter, Bannister, LawrenceH.; Berry Martin M.; Collins; Patricia, Mary Dyson; Dussek, Julien E.; Feruson, Mark W.J.; Gray's Anatomy, The Anatomical Basis Of Medicine and Surgery, $38^{\text {th }}$ edition, Ch-10, Pg $1572-1574$.

[5] Keith L. Moore, Arthur F. Dalley Clinically Oriented Anatomy $5^{\text {th }}$ edition, Ch -5 Pg- $670-671$.

[6] Vishram Singh Anatomy Of Abdomen \& Lower Limb, Elservier, Ch - 27, Pg- 422-427.

[7] Brearley S, Simms MH, Shearman CP. Peripheral pulse palpation: an unreliable physical sign. Ann R Coll Surg Eng 1992; 169-71.

[8] Gross DE, de Trafford JC, Roberts VC et al. Raise ankle/brachial index in insulin treated diabetic patients. Diabet Med 1989;6:5768. 